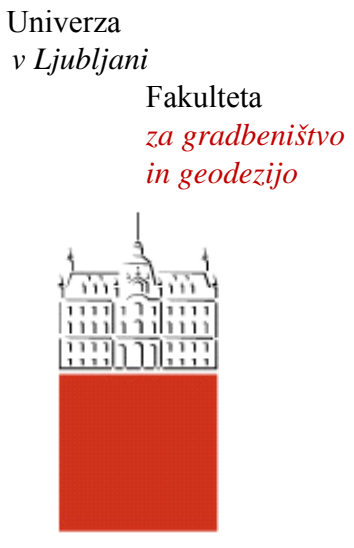

Jamova cesta 2

1000 Ljubljana, Slovenija

http://www3.fgg.uni-lj.si/

\section{DRUGG - Digitalni repozitorij UL FGG http://drugg.fgg.uni-lj.si/}

Ta članek je avtorjeva zadnja recenzirana različica, kot je bila sprejeta po opravljeni recenziji.

Prosimo, da se pri navajanju sklicujte na bibliografske podatke, kot je navedeno:
University
of Ljubljana

Faculty of

Civil and Geodetic

Engineering

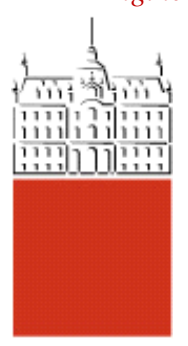

Jamova cesta 2

SI - 1000 Ljubljana, Slovenia

http://www3.fgg.uni-lj.si/en/

DRUGG - The Digital Repository http://drugg.fgg.uni-lj.si/

This version of the article is author's manuscript as accepted for publishing after the review process.

When citing, please refer to the publisher's bibliographic information as follows:

Gams, M., Planinc, I., Saje, M. 2007. Energy conserving time integration scheme for geometrically exact beam. Computer Methods in Applied Mechanics and Engineering 196,17-20: 2117-2129 DOI: 10.1016/j.cma.2006.10.012. 


\title{
Energy conserving time integration scheme for geometrically exact beam
}

\author{
M. Gams, I. Planinc and M. Saje \\ University of Ljubljana, Faculty of Civil and Geodetic Engineering, Jamova 2, \\ SI-1115 Ljubljana, Slovenia
}

\begin{abstract}
An energy conserving finite-element formulation for the dynamic analysis of geometrically non-linear beam-like structures undergoing large overall motions has been developed. The formulation is based on classical displacement-based planar beam finite elements described in an inertial frame. It takes into account finite axial, bending and shear strains. A theoretically consistent approach is used to derive a novel and simple energy conserving scheme, which is distinct in that the unconventional, a finite-size incremental strain update is used rather than the update in the strong form. Numerical examples demonstrate perfect energy conservation, stability and robustness of the scheme, and good convergence properties in terms of both the Newton-Raphson method and time step size.
\end{abstract}

Key words: non-linear dynamics, geometrically exact beam, stiff problems, time integration, conserving algorithms 


\section{Introduction}

Non-linear dynamics of engineering structures is often described by the stiff system of differential equations. Such equations arise due to the presence of large difference in stiffness among different components of a structure, and/or difference in deformation modes, and when the Lagrange multiplier technique is employed to impose the internal or external kinematic constraints. The classical Newmark time integration scheme [29] and many others are conditionally stable for non-linear systems and are hence not appropriate for such systems. Instable behaviour of these schemes when applied to stiff structural systems has been often experienced in practice and is well documented, see, e.g. $[15,17,21,24]$.

There are several approaches to overcome the instability problems in the analysis of stiff structural systems. A very popular approach is to introduce an artificial dissipation of energy into the system, which stabilizes the dynamic response of the system, but its energy is diminishing with time even in conservative systems. Such schemes are clearly not appropriate for the analysis of a long-term dynamic response. One of the earliest energy decaying schemes was the Hilbert-Hughes-Taylor (HHT) scheme [19]. The scheme was primarily introduced to eliminate high frequency oscillations of a pure numerical origin resulting typically in the use of the Newmark scheme. Its generalization, HHT- $\alpha$ scheme [13], provides an even better compromise between accuracy and stability requirements. These two schemes unconditionally dissipate energy in linear systems and often, but not always, yield satisfactory results in non-linear systems [17]. When applied to non-linear problems, energy can be created in a time step and not dissipated [3,15], which leads to potential 
instabilities. An alternative strategy to introduce controllable numerical dissipation, denoted as 'generalized energy-momentum method', was presented and applied to 3D trusses by Kuhl and Crisfield [26] and to shells by Kuhl and Ramm [27]. This method represents the combination of the generalized HHT- $\alpha$ method and the energy-momentum method of Simo and Tarnow [37]. Recent investigations in time-integration schemes with energy dissipation are developed within the framework of the time-discontinous Galerkin method $[4,5,9,10]$. Using a finite difference scheme, Armero and Romero [1,2] have obtained an energy dissipative integrator with a controllable numerical dissipation in the high frequency range. Goicolea and Orden [18] employed the discrete derivative concept for integrating non-linear Hamiltonian systems in conjunction with the penalty method to enforce the constraints. An outstanding performance in terms of robustness and accuracy was reported. A very different energy dissipating approach was proposed by Romero and Armero [31] and extended to 3D beams by Ibrahimbegović and Mamouri [22] who introduced specific algorithmic constitutive equations with artificial dissipating parts. The degree of artificial dissipation can be controlled by parameter $\alpha$. These schemes are analytically proven to dissipate energy in non-linear systems, which is a formal proof of the unconditional stability of the scheme. Bottasso et al. [11] showed how the Runge-Kutta method can be employed to design decaying schemes for non-linear dynamics. Another family of time integrators for solving the stiff equations of motion of classical mechanics in a general Hamiltonian context is based on the time-continuous Galerkin method $[7,12]$.

An alternative approach is the energy conserving scheme. Such a scheme is unconditionally stable [20], but often very high, unrealistic frequency oscillations 
are observed in the dynamic response, which can hinder the convergence of the Newton method for the solution of the non-linear algebraic equations, see e.g. [3]. The earliest schemes employing the energy conserving approach were proposed by Simo et al. [35-37]. In the present work, a new energy conserving algorithm for the numerical time integration of non-linear structural systems is developed, which conserves both energy and linear and angular momenta, in exact and discrete form, and satisfies the kinematic constraints in the weak form. As in the energy conserving algorithms proposed by Simo et al. [36,37], Ibrahimbegović et al. [21-23], Crisfield and Shi [14] and several others, the present algorithm employs the midpoint time integration rule. The essential step in the construction of the present algorithm is the derivation showing that the strain update should be made in an incremental way, if we desire to obtain automatically a zero energy increment in a time step. This algorithm can easily be modified to introduce a controllable energy decay using the algorithmic constitutive equations with artificial dissipation [22,31]. This line of development will, however, not be followed in the present paper.

In order to place the algorithm into the very applicable multibody and structural engineering context, the deduction is presented only for the case of the classical, displacement-based geometrically exact planar beam finite element formulation [35]. The generalization of the algorithm to other types of structural systems like 3D beams, plates and shells is a straightforward matter, as both ideology and technology remain the same. In fact, the majority of the time integrators for stiff systems have been derived in the context of the beam-like structures. Crisfield and Shi [14] proposed the midpoint based energy conserving integrator for their co-rotational planar truss formulation. Crisfield et al. [15] discussed various endpoint and midpoint time integra- 
tion algorithms for the dynamic analysis of their co-rotational spatial beam. They showed that their proposed midpoint scheme can be considered as an 'approximately energy conserving algorithm'. They also introduced a method with the numerical damping. Stander and Stein [38] studied the planar beam element proposed by Simo and Vu-Quoc [35] by imposing an explicit constantenergy constraint resulting in energy conservation. Jelenić and Crisfield [24] extended their master-slave formulation for 3D beams and joints to dynamic problems using the midpoint formulation, which lead to conservation of both energy and momenta. Ibrahimbegović and Mamouri [21,22] used a modified, non-linear, unconventional rotational velocity and torque updates in such a way that the scheme conserves energy. Sansour et al. [33,34] developed an energy-momentum conserving scheme applicable to any shell theory and any non-linear form of strain-displacement relations. The scheme was very successfully applied in the finite element analysis of dynamics of their seven degree of freedom shell theory. Regarding the non-linear beams as constrained mechanical systems from the outset and employing the Hamiltonian formulation of the semi-discrete beam, Betsch and Steinmann [8] derived an energy conserving scheme, which is both accurate and stable, and does not lead to spurious oscillations in the stress resultants. The main drawback of their approach seems to be the presence of the Lagrange multipliers leading to a greater number of unknowns than in other formulations. While the specific measures taken by any of the above cited authors are different, they all prove energy conservation analytically, in an explicit way, or numerically, which is a sufficient condition for the stability of the integrator.

When the Lagrange multipliers are used to impose the kinematic constraints, the governing dynamical equations become differential-algebraic equations. 
The frequencies associated with the algebraic equations are infinite, which makes the system stiff by definition. One such example is the strain-based finite element formulation for 2D geometrically exact beams by Gams et al. [16], and a number of approaches have been proposed for the integration, see, e.g. [3-6,8-11,18,23]. We do not discuss such constrained dynamical systems in the present paper, however.

The outline of the paper is as follows. In the next section, we present the governing equations of the dynamics of the geometrically exact Reissner's [30] planar beam, undergoing large overall planar motion. Various continuum and time-discrete forms of the Hamilton principle and the strong and weak forms of the kinematic constraints are given there. The central part of the paper is Section 2.6, where the energy conserving scheme is derived and its conservation properties proved. The scheme is distinct in that the unconventional, a finite-size incremental strain update is used, implied by the strict distinction between the infinitesimal and the finite-change operator. Consequently, the proposed scheme is somewhat more complex to implement. Because the automated code generation software package AceGen [25] was employed to generate the finite-element matrices needed for the computer code, this presented no additional complication. Section 3 describes the displacement-based finite-element implementation of the proposed scheme. In Section 4, we present numerical examples and comments. We show that the present energy conserving scheme perfectly conserves energy. The paper ends with some conclusions. 


\section{Construction of an energy conserving scheme for the dynamics of a non-linear beam}

\subsection{Planar Reissner's beam model [30]}

We consider the deformation of an initially straight planar elastic beam of initial length $L$ in the $(x, y)$-plane of a spatial Cartesian coordinate system $(x$, $y, z)$ with base vectors $\boldsymbol{e}_{x}, \boldsymbol{e}_{y}$ and $\boldsymbol{e}_{z}$ (see Fig. 1). The beam is initially rotated with respect to $\boldsymbol{e}_{x}$ by an angle $\varphi_{0}$. A material point on the beam centroid axis is identified by the material coordinate, $s \in[0, L]$. The cross-sections, associated with the material points, are assumed constant and symmetric with respect to the plane $(x, y)$. The beam is subjected to time dependent distributed loads $p_{x}(s, t), p_{y}(s, t)$ and $m_{z}(s, t)$, measured per unit length of the undeformed axis, and generalized point loads $S_{k}(t)(k=1,2, \ldots, 6)$ at its ends. Loads are assumed to be deformation-independent. Membrane, shear and bending strains are taken into account.

\subsection{Kinematic constraints}

The spatial position of an arbitrary material point of the centroid axis of the beam in the deformed configuration at $t>0$ is described by the position vector $\boldsymbol{r}(s, t)$

$$
\boldsymbol{r}(s, t)=(x(s)+u(s, t)) \boldsymbol{e}_{x}+(y(s)+v(s, t)) \boldsymbol{e}_{x}
$$

where $x(s)$ and $y(s)$ are the initial $x$ and $y$ coordinates of the material point, identified by ' $s$ ', and $u(s, t)$ and $v(s, t)$ are its $x$ and $y$ displacement components 

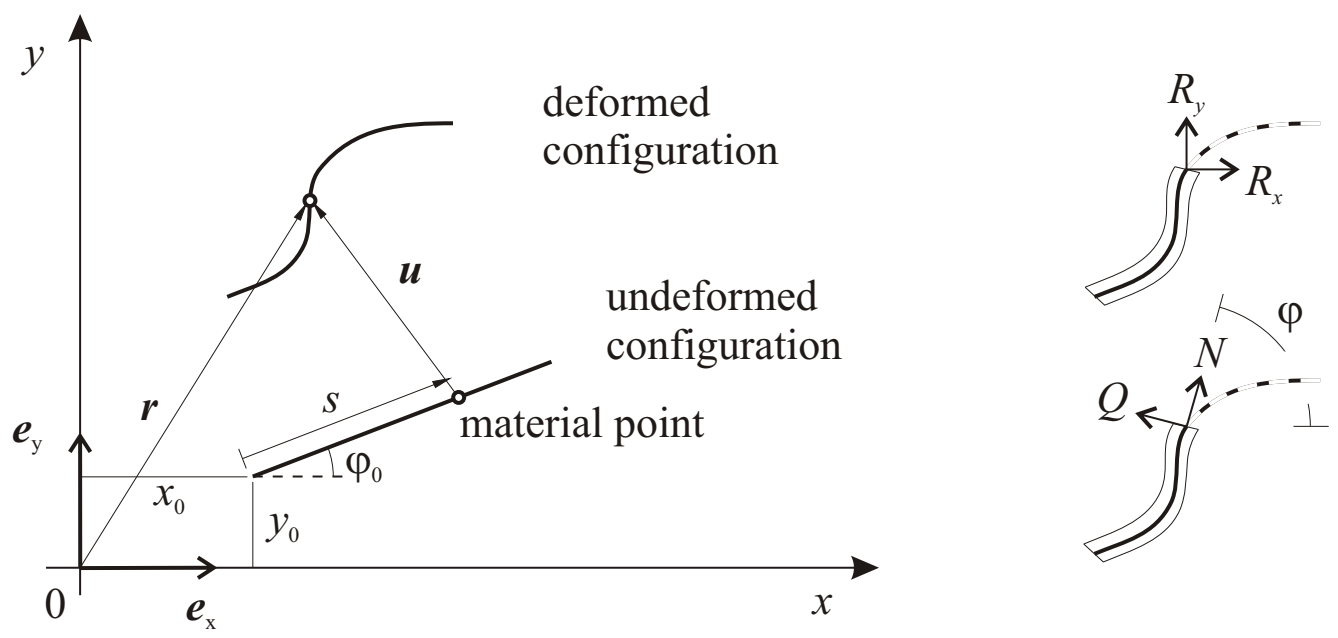

Fig. 1. Plane beam, in undeformed and deformed configurations, left. Notation of stress resultants, right.

at time $t$, respectively. As the beam is assumed straight in the undeformed configuration, the initial coordinates of a generic material point of the centroid axis are simply $(x, y)=\left(x_{0}+s \cos \varphi_{0}, y_{0}+s \sin \varphi_{0}\right)$, with $x_{0}$ and $y_{0}$ being the coordinates of the boundary material point $s=0$ (Fig. 1). The kinematic constraints of the geometrically exact beam theory have been provided by Reissner [30]; they read:

$$
\begin{gathered}
1+\varepsilon=\left(x^{\prime}+u^{\prime}\right) \cos \varphi+\left(y^{\prime}+v^{\prime}\right) \sin \varphi \\
\gamma=-\left(x^{\prime}+u^{\prime}\right) \sin \varphi+\left(y^{\prime}+v^{\prime}\right) \cos \varphi \\
\kappa=\varphi^{\prime} .
\end{gathered}
$$

In Eqs. (2)-(4), the prime (') denotes the derivative with respect to $s$, whereas functions $\varepsilon(s, t)>-1, \gamma(s, t), \kappa(s, t)$ and $\varphi(s, t)$ denote the extensional strain, the shear strain, the bending strain and the rotation of the cross-section, respectively. 


\subsection{Principle of virtual work}

Given at time $t$, the standard principle of virtual work for the dynamics of a planar beam states:

$$
\begin{gathered}
\int_{0}^{L}(N \delta \varepsilon+Q \delta \gamma+M \delta \kappa) \mathrm{d} s- \\
\int_{0}^{L}\left[\left(p_{x}-A \rho \ddot{u}\right) \delta u+\left(p_{y}-A \rho \ddot{v}\right) \delta v+\left(m_{z}-I \rho \ddot{\varphi}\right) \delta \varphi\right] \mathrm{d} s- \\
\sum_{k=1}^{6} S_{k} \delta W_{k}=0 .
\end{gathered}
$$

Here $N, Q$ and $M$ are the cross-sectional stress resultants. For a linear elastic material, they are assumed to be linear functions of strains

$$
\begin{aligned}
& N=E A \varepsilon, \\
& Q=G A_{\mathrm{S}} \gamma, \\
& M=E I \kappa .
\end{aligned}
$$

$E$ and $G$ are elastic and shear moduli, $A$ and $A_{\mathrm{S}}$ are the area and the shear area of the cross-section of the beam, and $I$ is its moment of inertia; $\rho$ is the density of material; $\delta u, \delta v$ and $\delta \varphi$ are virtual displacements and rotation; $\delta W_{k}$ $(k=1,2, \ldots, 6)$ are the generalized boundary virtual displacements [32]. The superposed dot denotes the differentiation with respect to time.

Functions $\delta \varepsilon, \delta \gamma$ and $\delta \kappa$ are virtual strains. Their relations to $\delta u, \delta v$ and $\delta \varphi$ are obtained by the variation of the kinematic constraints, Eqs. (2)-(4), and read

$$
\begin{gathered}
\delta \varepsilon=\delta u^{\prime} \cos \varphi+\delta v^{\prime} \sin \varphi+\delta \varphi\left(-\left(x^{\prime}+u^{\prime}\right) \sin \varphi+\left(y^{\prime}+v^{\prime}\right) \cos \varphi\right) \\
\delta \gamma=-\delta u^{\prime} \sin \varphi+\delta v^{\prime} \cos \varphi+\delta \varphi\left(-\left(x^{\prime}+u^{\prime}\right) \cos \varphi+\left(y^{\prime}+v^{\prime}\right) \sin \varphi\right) \\
\delta \kappa=\delta \varphi^{\prime}
\end{gathered}
$$


These equations will play an important role in devising the energy conserving scheme. Substituting virtual strains $\delta \varepsilon, \delta \gamma$ and $\delta \kappa$ from Eqs. (7)-(9) into Eq. (5) gives

$$
\begin{gathered}
\int_{0}^{L}\left[R_{x} \delta u^{\prime}+R_{y} \delta v^{\prime}+\left(-\left(x^{\prime}+u^{\prime}\right) R_{y}+\left(y^{\prime}+v^{\prime}\right) R_{x}\right) \delta \varphi+M \delta \varphi^{\prime}\right] \mathrm{d} s- \\
\int_{0}^{L}\left[\left(p_{x}-A \rho \ddot{u}\right) \delta u+\left(p_{y}-A \rho \ddot{v}\right) \delta v+\left(m_{z}-I \rho \ddot{\varphi}\right) \delta \varphi\right] \mathrm{d} s- \\
\sum_{k=1}^{6} S_{k} \delta W_{k}=0 .
\end{gathered}
$$

Here, $R_{x}$ and $R_{y}$ are the cross-sectional stress resultants with respect to the spatial basis (see Fig. 1):

$$
R_{x}=N \cos \varphi-Q \sin \varphi, \quad R_{y}=N \sin \varphi+Q \cos \varphi
$$

The terms $R_{x} \delta u^{\prime}, R_{y} \delta v^{\prime}$ and $M \delta \varphi^{\prime}$ in Eq. (10) are integrated by parts, resulting in

$$
\begin{gathered}
\int_{0}^{L}\left[\left(R_{x}^{\prime}+p_{x}-A \rho \ddot{u}\right) \delta u+\left(R_{y}^{\prime}+p_{y}-A \rho \ddot{v}\right) \delta v+\right. \\
\left.\left(M^{\prime}+\left(x^{\prime}+u^{\prime}\right) R_{y}-\left(y^{\prime}+v^{\prime}\right) R_{x}+m_{z}-I \rho \ddot{\varphi}\right) \delta \varphi\right] \mathrm{d} s+ \\
\left(S_{1}+R_{x}(0)\right) \delta W_{1}+\left(S_{2}+R_{y}(0)\right) \delta W_{2}+\left(S_{3}+M(0)\right) \delta W_{3}+ \\
\left(S_{4}-R_{x}(L)\right) \delta W_{4}+\left(S_{5}-R_{y}(L)\right) \delta W_{5}+\left(S_{6}-M(L)\right) \delta W_{6}=0 .
\end{gathered}
$$

In the construction of the finite-element solution, the form given in Eq. (10) rather than this modified principle of virtual work is employed. 


\subsection{Hamilton's principle and the weak form of constraints}

The next step consists of integrating in time the modified virtual work principle, Eq. (12), over the time interval $\left[t_{n}, t_{n+1}\right]$

$$
\begin{gathered}
\int_{t_{n}}^{t_{n+1}}\left[\int _ { 0 } ^ { L } \left(\left(R_{x}^{\prime}+p_{x}-A \rho \ddot{u}\right) \delta u+\left(R_{y}^{\prime}+p_{y}-A \rho \ddot{v}\right) \delta v+\right.\right. \\
\left.\left(M^{\prime}+\left(x^{\prime}+u^{\prime}\right) R_{y}-\left(y^{\prime}+v^{\prime}\right) R_{x}+m_{z}-I \rho \ddot{\varphi}\right) \delta \varphi\right) \mathrm{d} s+ \\
\left(S_{1}+R_{x}(0)\right) \delta W_{1}+\left(S_{2}+R_{y}(0)\right) \delta W_{2}+\left(S_{3}+M(0)\right) \delta W_{3}+ \\
\left.\left(S_{4}-R_{x}(L)\right) \delta W_{4}+\left(S_{5}-R_{y}(L)\right) \delta W_{5}+\left(S_{6}-M(L)\right) \delta W_{6}\right] \mathrm{d} t=0 .
\end{gathered}
$$

Eq. (13) represents Hamilton's principle of Reissner's beam model. The time integration in Eq. (13) will be performed numerically. In order to apply the numerical time integration in a consistent way both for the principle and its constraining kinematic and constitutive equations, we first differentiate the constraining equations with respect to time. The time derivatives of the kinematic equations, Eqs. (2)-(4), read

$$
\begin{gathered}
\dot{\varepsilon}=\dot{u}^{\prime} \cos \varphi+\dot{v}^{\prime} \sin \varphi+\dot{\varphi}\left(-\left(x^{\prime}+u^{\prime}\right) \sin \varphi+\left(y^{\prime}+v^{\prime}\right) \cos \varphi\right), \\
\dot{\gamma}=-\dot{u}^{\prime} \sin \varphi+\dot{v}^{\prime} \cos \varphi+\dot{\varphi}\left(-\left(x^{\prime}+u^{\prime}\right) \cos \varphi-\left(y^{\prime}+v^{\prime}\right) \sin \varphi\right), \\
\dot{\kappa}=\dot{\varphi}^{\prime} .
\end{gathered}
$$

The time derivatives of the constitutive equations, Eq. (6), are:

$$
\begin{aligned}
& \dot{N}=E A \dot{\varepsilon}, \\
& \dot{Q}=G A_{\mathrm{S}} \dot{\gamma}, \\
& \dot{M}=E I \dot{\kappa} .
\end{aligned}
$$




\subsection{Time discretization}

For the time discretization of Hamilton's principle, Eq. (13), and its adjoined set of the constraining kinematic and constitutive equations, Eqs. (14) and (15), we use the following set of 'midpoint' approximation rules:

$$
\begin{gathered}
\int_{t_{n}}^{t_{n+1}} f(t) \mathrm{d} t=f\left(t_{m}\right) \Delta t, \\
t_{m}=\frac{1}{2}\left(t_{n}+t_{n+1}\right), \\
\Delta t=t_{n+1}-t_{n}, \\
\dot{f}_{m}=\frac{f_{n+1}-f_{n}}{\Delta t}=\frac{\Delta f}{\Delta t}, \\
\ddot{f}_{m}=\frac{\dot{f}_{n+1}-\dot{f}_{n}}{\Delta t}=\frac{\Delta \dot{f}}{\Delta t} .
\end{gathered}
$$

' $f$ ' refers to an arbitrary function of $t$. Subscript ' $m$ ' refers to the midpoint configuration at $t_{m}=t_{n}+\frac{1}{2} \Delta t$, ' $n$ ' to a time station at $t_{n}$ and ' $n+1$ ' to $t_{n+1}=t_{n}+\Delta t$

The application of the midpoint rule to Hamilton's principle (13) yields

$$
\begin{gathered}
\Delta t\left[\int _ { 0 } ^ { L } \left(\left(R_{x}^{\prime}+p_{x}-A \rho \ddot{u}\right)_{m} \delta u_{m}+\left(R_{y}^{\prime}+p_{y}-A \rho \ddot{v}\right)_{m} \delta v_{m}+\right.\right. \\
\left.\left(M^{\prime}+\left(x^{\prime}+u^{\prime}\right) R_{y}-\left(y^{\prime}+v^{\prime}\right) R_{x}+m_{z}-I \rho \ddot{\varphi}\right)_{m} \delta \varphi_{m}\right) \mathrm{d} s+ \\
\left(S_{1}+R_{x}(0)\right)_{m} \delta W_{1 m}+\left(S_{2}+R_{y}(0)\right)_{m} \delta W_{2 m}+\left(S_{3}+M(0)\right)_{m} \delta W_{3 m}+ \\
\left.\left(S_{4}-R_{x}(L)\right)_{m} \delta W_{4 m}+\left(S_{5}-R_{y}(L)\right)_{m} \delta W_{5 m}+\left(S_{6}-M(L)\right)_{m} \delta W_{6 m}\right]=0 .
\end{gathered}
$$

After cancelling $\Delta t$, we can extract equations corresponding to the individual variations and obtain the dynamic equilibrium equations for $s \in[0, L], t \in$ $\left[t_{n}, t_{n+1}\right]:$

$$
\left(R_{x}^{\prime}+p_{x}-A \rho \ddot{u}\right)_{m}=0,
$$




$$
\begin{gathered}
\left(R_{y}^{\prime}+p_{y}-A \rho \ddot{v}\right)_{m}=0 \\
\left(M^{\prime}+\left(x^{\prime}+u^{\prime}\right) R_{y}-\left(y^{\prime}+v^{\prime}\right) R_{x}+m_{z}-I \rho \ddot{\varphi}\right)_{m}=0
\end{gathered}
$$

and the related boundary conditions at $s=0$ and $s=L$ :

$$
\begin{aligned}
& \left(S_{1}+R_{x}(0)\right)_{m}=0, \quad\left(S_{2}+R_{y}(0)\right)_{m}=0, \quad\left(S_{3}+M(0)\right)_{m}=0, \\
& \left(S_{4}-R_{x}(L)\right)_{m}=0, \quad\left(S_{5}-R_{y}(L)\right)_{m}=0, \quad\left(S_{6}-M(L)\right)_{m}=0 .
\end{aligned}
$$

The kinematic equations (14) are also numerically integrated in time by the midpoint rule yielding

$$
\begin{gathered}
\int_{t_{n}}^{t_{n+1}} \dot{\varepsilon} \mathrm{d} t=\dot{\varepsilon}_{m} \Delta t=\Delta \varepsilon=\varepsilon_{n+1}-\varepsilon_{n} \\
=\Delta u^{\prime} \cos \varphi_{m}+\Delta v^{\prime} \sin \varphi_{m}+\Delta \varphi\left(-\left(x^{\prime}+u_{m}^{\prime}\right) \sin \varphi_{m}+\left(y^{\prime}+v_{m}^{\prime}\right) \cos \varphi_{m}\right) \\
\int_{t_{n}}^{t_{n+1}} \dot{\gamma} \mathrm{d} t=\dot{\gamma}_{m} \Delta t=\Delta \gamma=\gamma_{n+1}-\gamma_{n} \\
=-\Delta u^{\prime} \sin \varphi_{m}+\Delta v^{\prime} \cos \varphi_{m}+\Delta \varphi\left(-\left(x^{\prime}+u_{m}^{\prime}\right) \cos \varphi_{m}-\left(y^{\prime}+v_{m}^{\prime}\right) \sin \varphi_{m}\right) \\
\int_{t_{n}}^{t_{n+1}} \dot{\kappa} \mathrm{d} t=\dot{\kappa}_{m} \Delta t=\Delta \kappa=\Delta \varphi^{\prime}
\end{gathered}
$$

where $\dot{u}_{m}^{\prime} \Delta t=\Delta u^{\prime}, \dot{v}_{m}^{\prime} \Delta t=\Delta v^{\prime}, \dot{\varphi}_{m}^{\prime} \Delta t=\Delta \varphi^{\prime}$ has been assumed in accord with Eq. (19). Note the formal equality of Eqs. (7)-(9) and (22)-(24) in all respects but one: the first set of equations deals with the variations of the strains, in contrast to the second set, which deals with the finite-time increments. We will take advantage of this equality when constructing the energy conserving scheme.

If the same sequence of steps is applied to the second form of the principle of virtual work, Eq. (10), the following alternative time-discretized form of 
Hamilton's principle is obtained

$$
\begin{gathered}
\int_{0}^{L}\left[R_{x m} \delta u_{m}^{\prime}+R_{y m} \delta v_{m}^{\prime}+\left(-\left(x^{\prime}+u_{m}^{\prime}\right) R_{y m}+\left(y^{\prime}+v_{m}^{\prime}\right) R_{x m}\right) \delta \varphi_{m}+M_{m} \delta \varphi_{m}^{\prime}\right] \mathrm{d} s- \\
\int_{0}^{L}\left[\left(p_{x m}-A \rho \ddot{u}_{m}\right) \delta u_{m}+\left(p_{y m}-A \rho \ddot{v}_{m}\right) \delta v_{m}+\left(m_{z m}-I \rho \ddot{\varphi}_{m}\right) \delta \varphi_{m}\right] \mathrm{d} s- \\
\sum_{k=1}^{6} S_{k m} \delta W_{k m}=0 .
\end{gathered}
$$

This equation will be needed when proving the conservation properties of the time-integration scheme.

\subsection{Energy conservation}

The mechanical energy of the unloaded beam at a specific time is the sum of the strain and kinetic energy

$$
\mathcal{E}=\frac{1}{2} \int_{0}^{L}\left(E A \varepsilon^{2}+G A_{\mathrm{S}} \gamma^{2}+E I \kappa^{2}\right) \mathrm{d} s+\frac{1}{2} \int_{0}^{L}\left(A \rho \dot{u}^{2}+A \rho \dot{v}^{2}+I \rho \dot{\varphi}^{2}\right) \mathrm{d} s
$$

The change of the energy between two consecutive time stations is

$$
\begin{gathered}
\Delta \mathcal{E}=\mathcal{E}_{n+1}-\mathcal{E}_{n}= \\
\frac{1}{2} \int_{0}^{L}\left[E A\left(\varepsilon_{n+1}^{2}-\varepsilon_{n}^{2}\right)+G A_{\mathrm{S}}\left(\gamma_{n+1}^{2}-\gamma_{n}^{2}\right)+E I\left(\kappa_{n+1}^{2}-\kappa_{n}^{2}\right)\right] \mathrm{d} s+ \\
\frac{1}{2} \int_{0}^{L}\left[A \rho\left(\dot{u}_{n+1}^{2}-\dot{u}_{n}^{2}\right)+A \rho\left(\dot{v}_{n+1}^{2}-\dot{v}_{n}^{2}\right)+I \rho\left(\dot{\varphi}_{n+1}^{2}-\dot{\varphi}_{n}^{2}\right)\right] \mathrm{d} s .
\end{gathered}
$$

We rewrite the differences of the squares of $\varepsilon$ and $\dot{u}$ in the following fashion:

$$
\begin{aligned}
& \frac{1}{2}\left(\varepsilon_{n+1}^{2}-\varepsilon_{n}^{2}\right)=\frac{\varepsilon_{n+1}+\varepsilon_{n}}{2}\left(\varepsilon_{n+1}-\varepsilon_{n}\right)=\varepsilon_{m} \Delta \varepsilon, \quad \varepsilon_{m}=\frac{\varepsilon_{n+1}+\varepsilon_{n}}{2}, \\
& \frac{1}{2}\left(\dot{u}_{n+1}^{2}-\dot{u}_{n}^{2}\right)=\frac{\dot{u}_{n+1}+\dot{u}_{n}}{2}\left(\dot{u}_{n+1}-\dot{u}_{n}\right)=\dot{u}_{m} \Delta \dot{u}, \quad \dot{u}_{m}=\frac{\dot{u}_{n+1}+\dot{u}_{n}}{2} .
\end{aligned}
$$

Similar expressions are written for the pairs $(\gamma, \dot{v})$ and $(\kappa, \dot{\varphi})$. The above form for $\varepsilon_{m}$ and $\dot{u}_{m}$ assumes the trapezoidal rule for determining the values of strains $\left(\varepsilon_{m}, \gamma_{m}, \kappa_{m}\right)$ and velocities $\left(\dot{u}_{m}, \dot{v}_{m}, \dot{\varphi}_{m}\right)$ at the midpoint time configurations. 
The velocities are further worked on using rules (19) and (20) in the following way:

$$
\dot{u}_{m} \Delta \dot{u}=\frac{\Delta u}{\Delta t} \ddot{u}_{m} \Delta t=\ddot{u}_{m} \Delta u .
$$

These modifications along with the relations for the midpoint stress resultants emerging from the numerical integration of Eqs. (15) by the midpoint rule

$$
N_{m}=E A \varepsilon_{m}, \quad Q_{m}=G A_{\mathrm{S}} \gamma_{m}, \quad M_{m}=E I \kappa_{m}
$$

lead to a new form for the change of energy:

$$
\begin{aligned}
& \Delta \mathcal{E}=\frac{1}{2} \int_{0}^{L}\left(N_{m} \Delta \varepsilon+Q_{m} \Delta \gamma+M_{m} \Delta \kappa\right) \mathrm{d} s+ \\
& \frac{1}{2} \int_{0}^{L}\left(A \rho \ddot{u}_{m} \Delta u+A \rho \ddot{v}_{m} \Delta v+I \rho \ddot{\varphi}_{m} \Delta \varphi\right) \mathrm{d} s .
\end{aligned}
$$

By substituting $\Delta \varepsilon, \Delta \gamma$ and $\Delta \kappa$ with the expressions given in Eqs. (22)-(24) and after employing Eqs. (11), we get

$$
\begin{gathered}
\Delta \mathcal{E}=\int_{0}^{L}\left[R_{x m} \Delta u^{\prime}+R_{y m} \Delta v^{\prime}+\right. \\
\left.\left(-\left(x^{\prime}+u_{m}^{\prime}\right) R_{y m}+\left(y^{\prime}+v_{m}^{\prime}\right) R_{x m}\right) \Delta \varphi+M_{m} \Delta \varphi^{\prime}\right] \mathrm{d} s+ \\
\int_{0}^{L}\left(A \rho \ddot{u}_{m} \Delta u+A \rho \ddot{v}_{m} \Delta v+I \rho \ddot{\varphi}_{m} \Delta \varphi\right) \mathrm{d} s .
\end{gathered}
$$

Note that the deduction yielding to the above equation is identical to the one leading to Eq. (10).

After we eliminate the external loading terms from Eq. (25) and compare the result to Eq. (30), we see that the expressions in equations are virtually the same, with the differences being that (i) in Eq. (25) we have infinitesimal variations, while in Eq. (30) there are finite-size changes in $u, v$ and $\varphi$; and (ii) Eq. (25) represents the principle of virtual work and thus identically equals to zero for any virtual generalized displacements, if the dynamic equilibrium equations are satisfied, while Eq. (30) only represents the change of 
energy at two consecutive time stations, which generally does not vanish for any finite-size increments of generalized displacements. If, however, the dynamic equilibrium equations and, consequently, the principle of virtual work are satisfied indeed, it is easy to show that Eq. (30) also vanishes for any finite increments $\Delta u, \Delta v, \Delta \varphi$, related to $\Delta \varepsilon, \Delta \gamma$ and $\Delta \kappa$ by Eqs. (22)-(24). Thus the energy in the time step is conserved.

Remark 1. It is significant that we have proven the conservation of the mechanical energy before the spatial discretization has been applied. Hence, the proposed scheme sets no restrictions whatsoever on the manner the equations are discretized in the space variable.

Remark 2. It is now rather obvious why we cannot fulfil the condition of the energy conservation solely using the basic form of the principle of virtual work as given in Eq. (5) and its constraints in the strong form (2)-(4).

Remark 3. The proposed time-integration scheme conserves energy only when computed for the discrete times, $t_{n+1}, t_{n}, \ldots$ Therefore, the conservation of energy holds true solely in a time-discrete, algorithmic sense. Similarly, the equations of the dynamic equilibrium are satisfied to be zero only at the midpoint times, at $t_{m}, t_{m+1}, \ldots$ Because the dynamic equilibrium equations are obtained as the time derivative of the linear and angular momenta, it follows that the linear and angular momenta are also conserved in an algorithmic sense during motion, yet at different time stations, i.e. at $t_{n}, t_{n+1}, \ldots$

Remark 4. The strong form of the kinematic equations is not exactly satisfied in our formulation. The drift from the kinematic constraint manifold depends mainly on the size of the time step. In most cases, the drift is so small that it can be neglected, see the discussion in our second numerical example. 
Remark 5. Unlike the formulations by, e.g. Ibrahimbegović and Mamouri [21] or Simo et al. [36], where the midpoint rotation matrix, $\Lambda_{m}$, is approximated by $\Lambda_{m}=\frac{1}{2}\left(\Lambda_{n}+\Lambda_{n+1}\right)$, which is not an orthogonal matrix, our formulation retains orthogonality of the rotation operator.

Remark 6 . Some energy conserving schemes are derived by heavily relying on substituting the linear operator $\delta$ with the finite-size incremental operator $\Delta$. This is not a consistent line of thinking, at least in the view of the authors of this article, and no such assumption has been made in our derivation. Nevertheless, we acknowledge the fact that, if we had also abused this substitution, we would have ended up with the same theoretical result as the one presented.

\section{Finite element formulation}

\subsection{Spatial and time discretization}

The displacement and rotation distributions along the beam axis are interpolated by the linear combination of the Lagrangian polynomials, $P_{i}(s)$, of an arbitrary order $k$. We denote the discrete nodal values of generalized displacements $(u, v$ and $\varphi)$ with their capital letter counterparts $\left(U_{i}, V_{i}\right.$ and $\Phi_{i}$, $i=1,2, \ldots, k)$. Equidistant points along the finite element axis in the undeformed configuration are used for the interpolation in spatial domain. Hence

$$
\begin{gathered}
u(s, t)=\sum_{i=1}^{k} U_{i}(t) P_{i}(s), \\
v(s, t)=\sum_{i=1}^{k} V_{i}(t) P_{i}(s), \\
\varphi(s, t)=\varphi_{0}+\sum_{i=1}^{k} \Phi_{i}(t) P_{i}(s) .
\end{gathered}
$$


The same type of interpolation rules are imployed to describe variations of virtual generalized displacements $(\delta u, \delta v, \delta \varphi)$, increments in generalized displacements $\left(\Delta u=u_{n+1}-u_{n}, \Delta w=w_{n+1}-w_{n}, \Delta \varphi=\varphi_{n+1}-\varphi_{n}\right)$ and time derivatives $(\dot{u}, \dot{v}, \dot{\varphi}, \ddot{u}, \ddot{v}, \ddot{\varphi})$. The derivatives with respect to $s$ are obtained by differentiating the interpolated variables. The time discretization employs the midpoint rule, which is applied to the discrete nodal values of displacements and rotations once the spatial discretization has been completed. To make the text shorter, we introduce a generic symbol $\Psi$, which stands for any of the discrete displacements $\left(U_{i}\right.$ or $V_{i}$ or $\left.\Phi_{i}\right)$, and present the midpoint rules as:

$$
\begin{gathered}
\Psi_{m}=\frac{\Psi_{n}+\Psi_{n+1}}{2}, \\
\dot{\Psi}_{m}=\frac{\Psi_{n+1}-\Psi_{n}}{\Delta t}, \\
\ddot{\Psi}_{m}=\frac{2}{\Delta t^{2}}\left(\Psi_{n+1}-\Psi_{n}-\dot{\Psi}_{n} \Delta t\right)
\end{gathered}
$$

along with the corresponding velocity update

$$
\dot{\Psi}_{n+1}=\frac{2}{\Delta t}\left(\Psi_{n+1}-\Psi_{n}-\frac{1}{2} \dot{\Psi}_{n} \Delta t\right)
$$

\subsection{Tangent stiffness matrix, mass matrix and residual vector}

The derivation of the tangent stiffness matrix of the displacement-based formulation in conjunction with the present time integration scheme can be a relatively tedious job to do, partly also due to the introduction of unconventional relations $(22)-(24)$. This is one of the reasons why we resorted to an automated code generation software package AceGen, developed by Korelc [25]. The package works inside the Mathematica [39] environment and can produce a finite element code in various programming languages. We used the package to generate the code for evaluating the tangent stiffness matrix, $K_{\mathrm{T}}$, 
the mass matrix, $M$, and the residual vector, $f_{\mathrm{R}}$. The code was then used in Matlab [28] to perform actual calculations. The main advantage of working in such an environment is that whenever we need to differentiate a functional, we just execute a command, which saves us a lot of time, guarantees the no-error results for the generated code and its computational optimization. The details of the energy conserving algorithm are schematically presented in Box 1 .

Box 1: Energy conserving scheme.

(1) Given nodal displacements and velocities at $t_{n}: \Psi_{n}, \dot{\Psi}_{n}$

1. Initialization: $\Psi_{n+1}:=\Psi_{n}, \dot{\Psi}_{n+1}:=\dot{\Psi}_{n}, \delta \Psi=0$

2. Newton's iteration

while $\frac{\|\delta \Psi\|}{\max (1,\|\Psi\|)}>$ tol

a. midpoint nodal displacements and velocities, Eqs. (34)-(36): $\Psi_{m}, \dot{\Psi}_{m}$

b. incremental nodal displacements: $\Delta \Psi=\Psi_{n+1}-\Psi_{n}$

c. incremental displacements, Eqs. (31)-(33): $\Delta u, \Delta v, \Delta \varphi$

d. midpoint displacements: $u_{m}, v_{m}, \varphi_{m}$

e. midpoint accelerations: $\ddot{u}_{m}, \ddot{v}_{m}, \ddot{\varphi}_{m}$

f. derivative of midpoint displacements with respect to $s: u_{m}^{\prime}, v_{m}^{\prime}, \varphi_{m}^{\prime}$

g. incremental strains, Eqs. (22)-(24): $\Delta \varepsilon, \Delta \gamma, \Delta \kappa$

h. strains: $\varepsilon_{n+1}=\varepsilon_{n}+\Delta \varepsilon, \gamma_{n+1}=\gamma_{n}+\Delta \gamma, \kappa_{n+1}=\kappa_{n}+\Delta \kappa$

i. midpoint forces, Eq. (28) and (11): $N_{m}, Q_{m}, M_{m} ; R_{x m}, R_{y m}$

j. residual vector: $\delta(\Gamma-\Sigma) \Rightarrow f_{\mathrm{R}}$

k. tangent stiffness matrix: $\delta^{2}(\Gamma-\Sigma) \Rightarrow K_{\mathrm{T}}, \quad K_{\mathrm{T}} \neq K_{\mathrm{T}}^{T}$

l. solve: $K_{\mathrm{T}} \delta \Psi=f_{\mathrm{R}} \Rightarrow \delta \Psi$

m. update: $\Psi_{n+1}:=\Psi_{n+1}+\delta \Psi$, Eq. (37): $\dot{\Psi}_{n+1}=\frac{2}{\Delta t}\left(\Psi_{n+1}-\Psi_{n}-\frac{1}{2} \dot{\Psi}_{n} \Delta t\right)$ end while

$\Gamma$ and $\Sigma$ in Box 1 designate the first and the second integrals in Eq. (25). When we neglect the effect of the external forces, Eq. (25) can be rewritten as

$$
\Gamma-\Sigma=0
$$

The integral in $\Gamma$ is numerically integrated by the reduced integration to alle- 
viate the locking, whereas the integral in $\Sigma$ is integrated fully. As observed in Box 1, the presented scheme is found fundamentally different when compared to other displacement-based finite element schemes, e.g. [3,14,21,22,36,38], because the weak kinematic equations (22)-(24) rather than their strong forms, Eqs. (2)-(4), are used to determine strains at $t_{n+1}$.

Remark 7 . Notice from Box 1 that the tangent stiffness matrix is not symmetric. The non-symmetry is a typical characteristic of energy conserving schemes, see, e.g. $[15,21,22,24]$.

Remark 8. Because the equations of a finite element have been derived in the spatial ('global') coordinate system, no further local-to-global transformation of the matrices is needed.

\subsection{An alternative 'strong' formulation}

Having to calculate strains repeatedly by the incremental Eqs. (22)-(24), which requires storing current strains in addition to the displacements, seems a bit uneconomical. This is overcome, if an alternative formulation is introduced, which exploits the direct evaluation of strains from current displacements using Eqs. (2)-(4) rather than the finite-size incremental relation (22)(24). Because time steps must be small by definition, this alternative way of evaluating strains at $t_{n+1}$ normally leads to only slightly different results for strains. The way these two variants of the formulation compute strains, suggests the terms 'weak' and 'strong' formulation, and this terminology will be adopted here. We will compare the results of these variants in the second numerical example. We wish to emphasize that the explicit theoretical proof of energy conservation only holds for the weak variant. If we wish to make the 
present strong formulation to be theoretically-based energy conserving one, we must construct a special angular velocity update and the related modified algorithmic constitutive equation for the bending moment, see, e.g. [21,22].

\section{Numerical examples}

\subsection{The swing pendulum}

This example, leading to a highly stiff system of differential equations, was originally proposed by Bauchau et al. [3,4], and subsequently analyzed by Ibrahimbegović and his co-workers [21-23]. The pendulum consists of a flexible beam hinged on both ends into two rigid links (Fig. 2). The rigid links impose a kinematic constraint corresponding to fixed distance between points $O_{1}$ and $A$, and $\mathrm{O}_{2}$ and $E$.



Fig. 2. The swing pendulum: geometry and load data.

A point mass $m=0.5 \mathrm{~kg}$ is rigidly connected to the flexible beam at its midspan. Point $B$ is the observation point. The material and geometric properties 
of the flexible beam are:

$$
\begin{gathered}
E=73 \cdot 10^{9} \mathrm{~N} / \mathrm{m}^{2}, A=0.05 \times 0.01 \mathrm{~m}^{2}, L=0.72 \mathrm{~m}, \\
I=\frac{1}{12} 0.05 \times 0.01^{3} \mathrm{~m}^{4}, \rho=2700 \mathrm{~kg} / \mathrm{m}^{3}, m_{\text {BEAM }}=0.972 \mathrm{~kg} .
\end{gathered}
$$

Shear strains are made negligible by setting a large value for the shear modulus $(G=100 E)$. As in [21] and [3] the rigid links are assumed weightless $(\rho=0$ $\mathrm{kg} / \mathrm{m}^{3}$ ) and their rigidity is modelled by assuming large Young's modulus, i.e. ten times the value of the modulus of the flexible beam.

The system is initially at rest. It is set in motion by a horizontal timedependent pulse at the mid-span of the beam. The time variation of the pulse intensity is shown in Fig. 2. After the pulse vanishes at $t=0.256 \mathrm{~s}$, the system is left to oscillate freely in such a way that the total energy is conserved.

Only the weak variant of the formulation is discussed in this example.

The sequence of deformed shapes of the swing pendulum are shown in Fig. 3. At first, the links are moving in the counter clock-wise direction. At roughly $t \approx 0.62 \mathrm{~s}$, the right link reverses its direction and starts moving clock-wise. Simultaneously, the horizontal velocity of the point mass changes its direction, which acts almost like an impact on the system. A smooth response in low frequencies abruptly changes into a complicated high frequency response. This event, however, does not perturb predominantly swinging motion.

In order to check if energy is conserved for any order of the spatial interpolation, we made use of three different orders of spatial interpolation, i.e. the linear, quadratic and cubic interpolation. The linear mesh employed 4 linear elements to model each of the rigid links and 40 linear elements for the flexible beam. The corresponding figures for the quadratic elements are 4 and 8 , and 


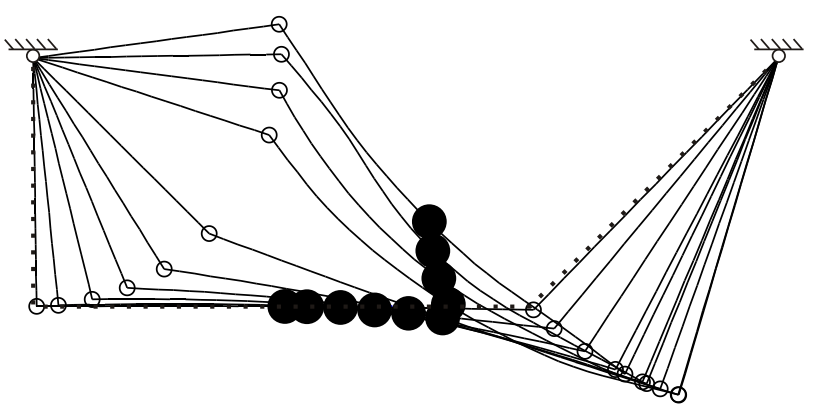

Fig. 3. Motion of the system, drawn until $1 \mathrm{~s}$, depicted in $0.1 \mathrm{~s}$ time intervals. The dotted line depicts the initial configuration. 16 quadratic elements, time step $\Delta t=0.0005 \mathrm{~s}$.

for the cubic elements 1 and 4 . We found out that the meshes with 48 linear, 16 quadratic or 6 cubic elements produced the results of nearly equal quality (Fig. 4).

To analyze the high frequency response of the beam in modes related to axial vibrations, we show the time variation of the axial force at the first Gauss point to the left of the midspan mass. We compare the results for three spatial interpolations in Fig. 5. As the locations of the first Gauss points in the three finite-element meshes are not coincident, these graphs should not be the same. Yet, the peak occurring at the time of the right link reversal is essentially the same for all three meshes. By contrast, the noise being produced afterwards is quite different. The least noise left-over is produced with quadratic elements. Any oscillations with such a high frequency could only be extremely poorly accounted for with the time step fixed to $0.0005 \mathrm{~s}$. Practically any oscillation modes with periods less than $0.005 \mathrm{~s}$ only produce noise. Nevertheless, the noise remains within the reasonable bounds, enforced by the energy conserving scheme.

We conclude this example with the graph of the total energy of the system (Fig. 
Displacement $[\mathrm{m}]$

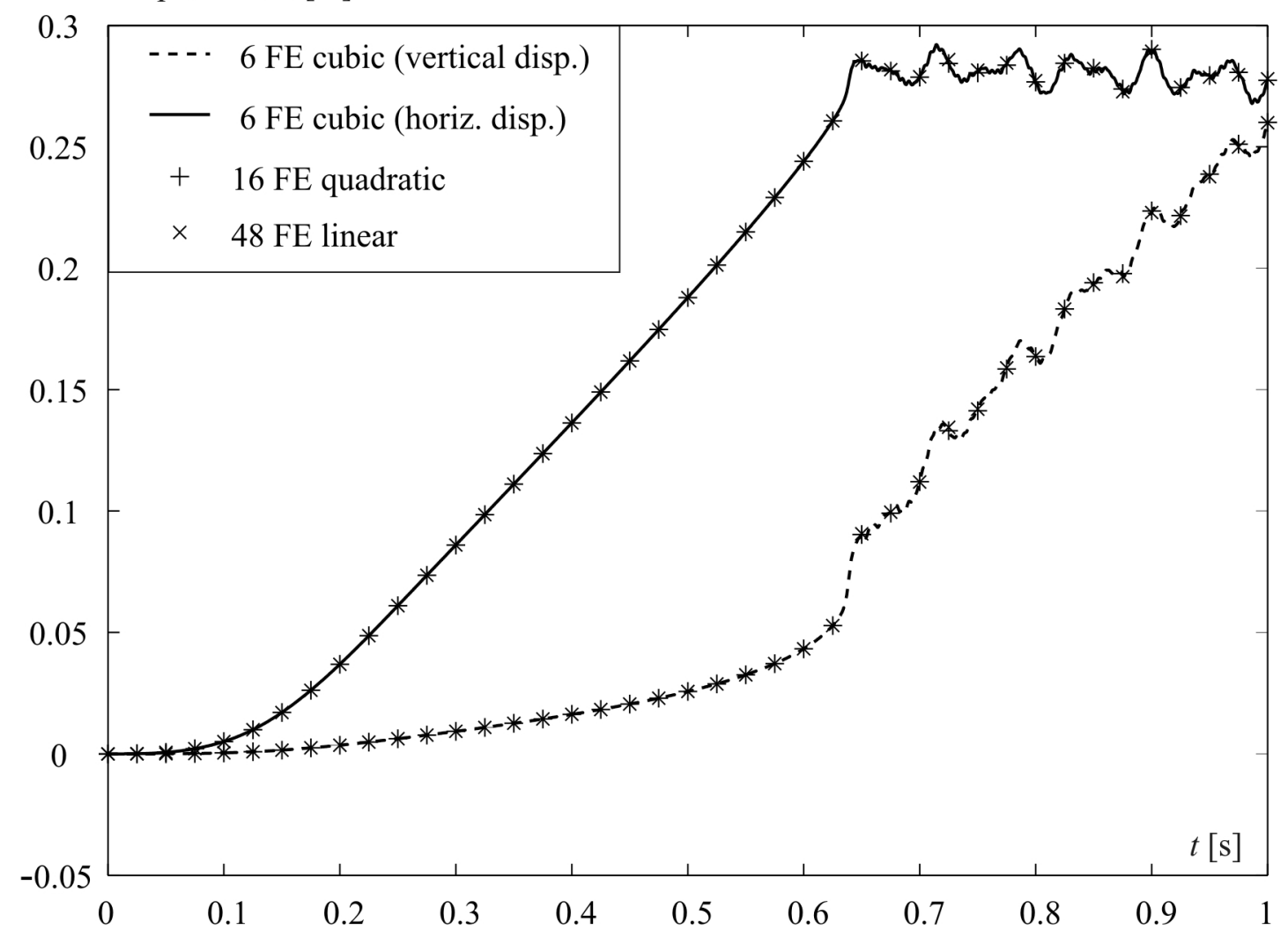

Fig. 4. Time variations of vertical and horizontal displacements at the point of observation, $B$. Comparison of results obtained by different spatial interpolations.

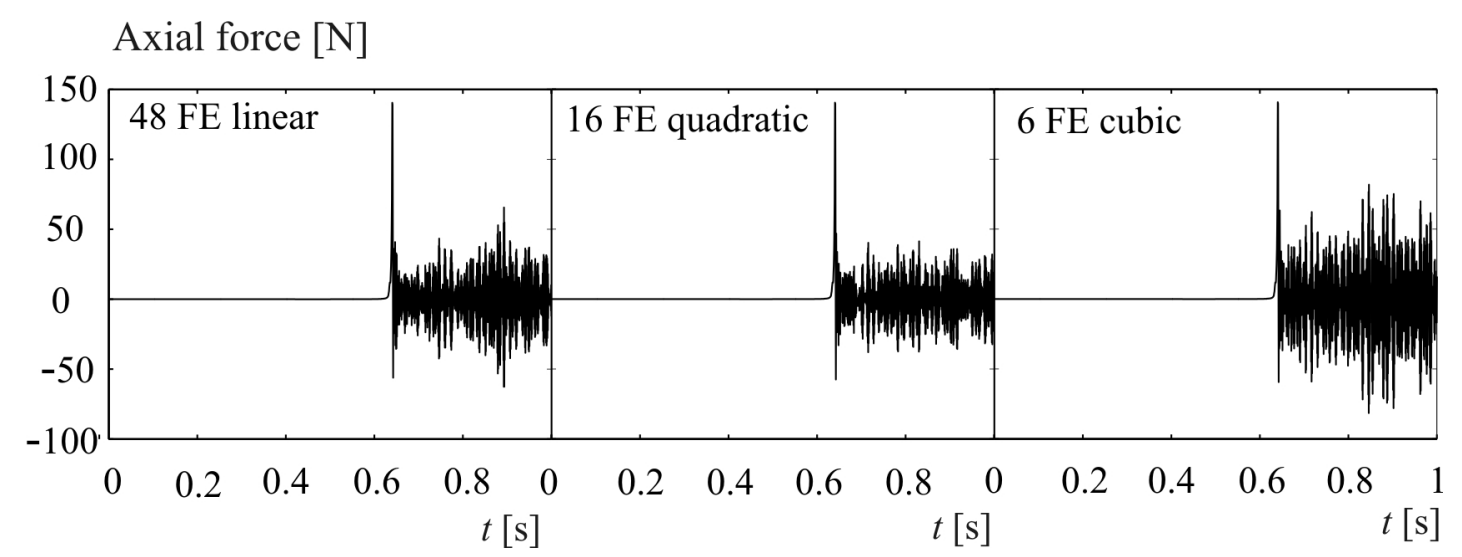

Fig. 5. Time variation of axial force at the first Gauss point to the left of the midspan mass. Comparison of different spatial interpolations. 
6). This particular graph was obtained by the use of the quadratic elements, but there is no difference whatsoever in graphs if any other order of the spatial interpolation has been employed.

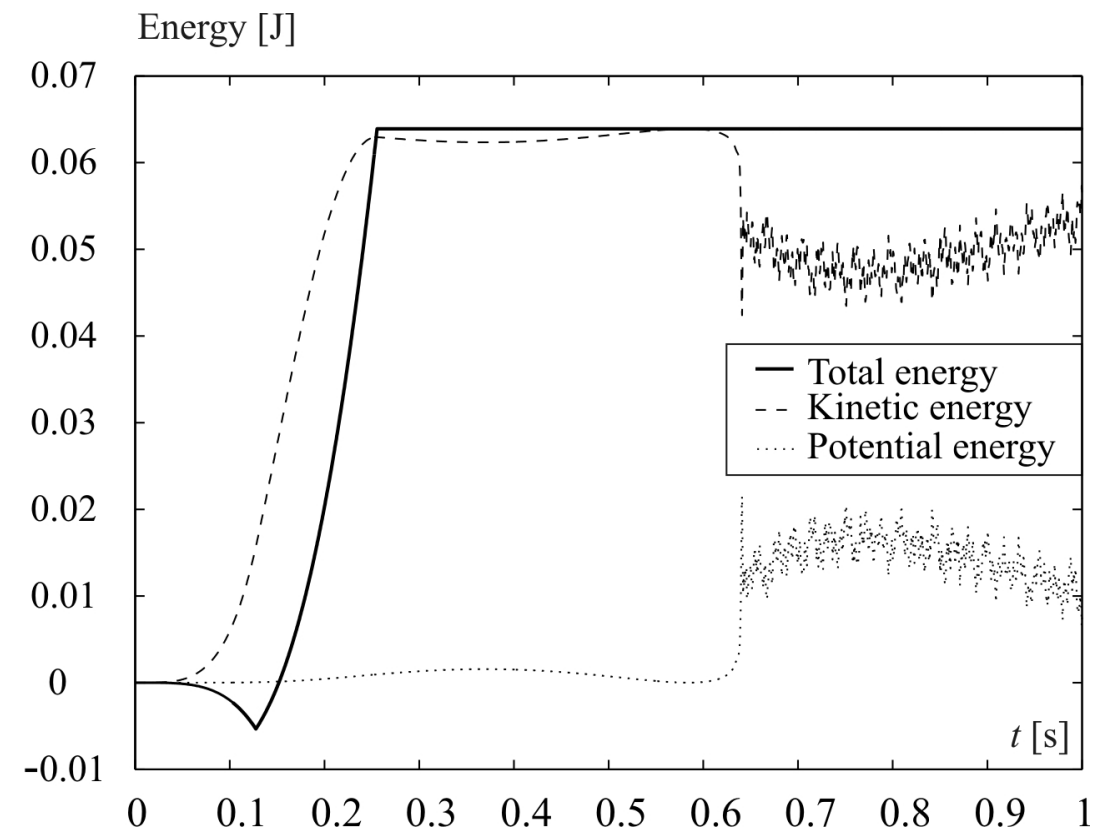

Fig. 6. Time variations of total, kinetic and potential energy of the system.

In an iterative solution of non-linear equations by a computer, the norm of the residual vector does not equal to zero in a strict mathematical sense of equality, and depends on the machine precision of the computer used and the tolerance required by the user. In the present study, the tolerance is set to bound the iterative increments in the Newton-Raphson procedure rather than the residual vector as

$$
\frac{\|\delta \Psi\|}{\max (1,\|\Psi\|)}<10^{-10}
$$

The inaccuracy is reflected in the non-constant values of the total mechanical energy with time. The flat line that we can observe after $0.256 \mathrm{~s}$, is, in fact, numerically a little bit curly. With the above given criterion being satisfied, we have observed the absolute difference between the maximum and the minimum 
energy within the time interval $t \in[0.256 \mathrm{~s}, 1 \mathrm{~s}]$ to be about $10^{-10}$ or less, see Table 1. This is considered to be a perfect energy conservation for double precision arithmetics.

The number of Newton's iterations in any time step ranged from 3 to 7 . In the majority of time steps, 4 to 5 iterations sufficed. This indicates that the high oscillations in axial forces do not slow down the convergence of the NewtonRaphson method.

Table 1

Maximum difference in total energy in time interval $t \in[0.256,1]$ for different spatial interpolations.

\begin{tabular}{ccc}
\hline \multicolumn{3}{c}{$\max ($ Energy $)-\min ($ Energy $)$} \\
\hline $48 \mathrm{FE} \mathrm{lin.}$ & $16 \mathrm{FE}$ quad. & $6 \mathrm{FE}$ cub. \\
\hline $1.6 \cdot 10^{-10}$ & $7.7 \cdot 10^{-11}$ & $1.4 \cdot 10^{-10}$ \\
\hline
\end{tabular}

\subsection{Planar motion of a multibody system}

This example was introduced by Ibrahimbegović and Mamouri [21] to demonstrate the versatility of their formulation for dealing with different types of joints. This also holds true for the present formulation, because, as in [21], the joints are accounted for through a simple condensation on the element level.

Moreover, the example is of interest in its own right, since it undergoes interesting configurations, responds in high and low frequencies, and cannot be satisfactorily solved with non-conserving methods, such as the Newmark [29] or HHT [19] methods, as was clearly shown in [21].

The multibody system under consideration is made of 4 flexible members interconnected by either revolute or prismatic joints (Fig. 7). Point $B$ is the 

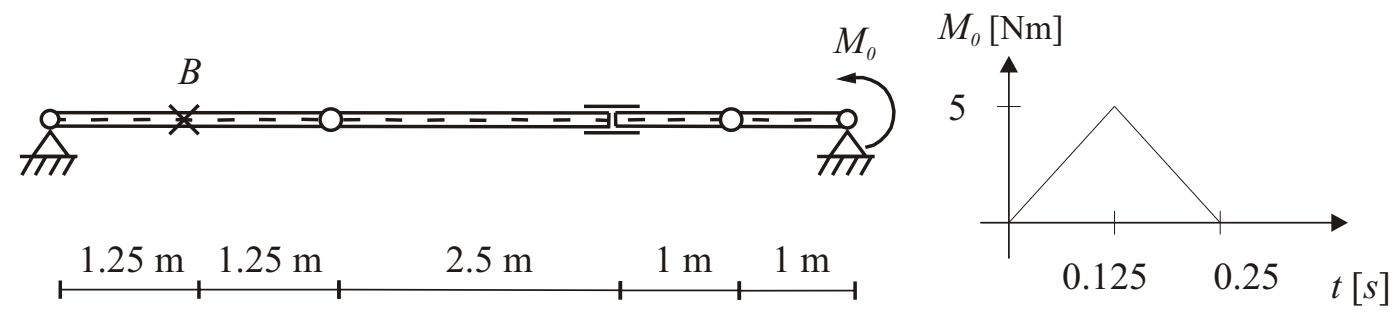

Fig. 7. The multibody system: geometry and load data.

observation point. The system is initially at rest. It is put into motion by a concentrated torque at the right support. The time variation of the torque is depicted in Fig. 7. The remaining descriptive data are:

$$
\begin{gathered}
E A=5.65 \cdot 10^{5} \mathrm{~N}, G A_{\mathrm{S}}=1.4038 \cdot 10^{5} \mathrm{~N}, E I=3.04 \cdot 10^{1} \mathrm{Nm}^{2}, \\
A \rho=1.35 \cdot 10^{-2} \mathrm{kgm}, I \rho=1.125 \cdot 10^{-6} \mathrm{kgm} .
\end{gathered}
$$

Again, three different spatial interpolations were used in combination with the weak version of the formulation. For a converged solution (i.e. a sufficiently accurate solution), at least 80 linear, 12 quadratic or 8 cubic elements were needed. The time variation of the vertical displacement at point $B$ is shown in Fig. 8.

The system exhibits a gradual increase towards a high frequency response. This is particularly true for the variation in time of the axial force at the first Gauss point to the left of point $B$, see Fig. 9 .

The results for the displacements and the axial force, if obtained by the strong version, compare well with the results obtained by the weak formulation, and the differences are so small that they are not visible on the graphs. Table 2 shows the absolute difference between the maximum and the minimum values of the total energy within the time interval $t \in[0.25 \mathrm{~s}, 1 \mathrm{~s}]$. The results clearly 


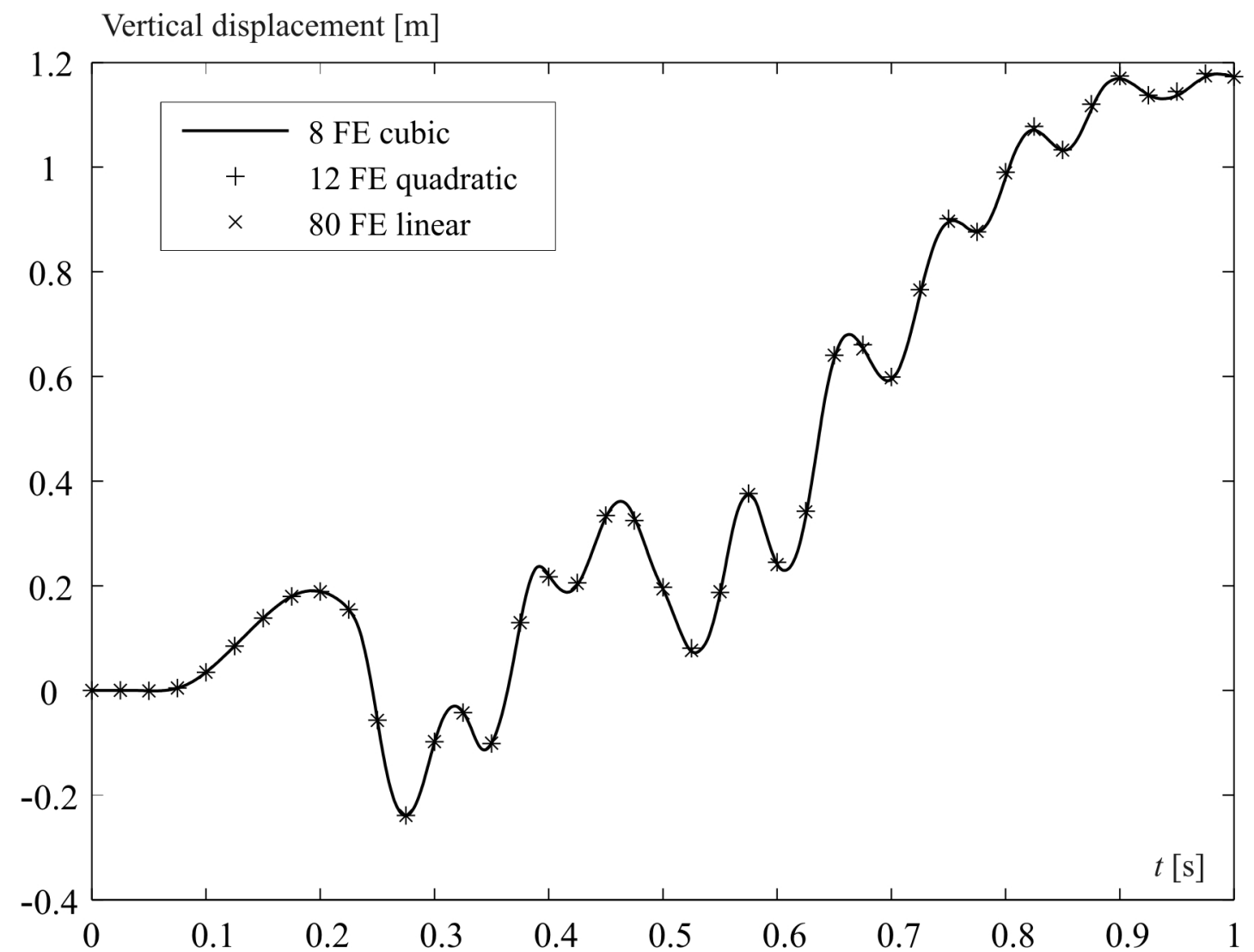

Fig. 8. Time variation of vertical displacement at the point of observation, $B$. Comparison of three different spatial interpolations.

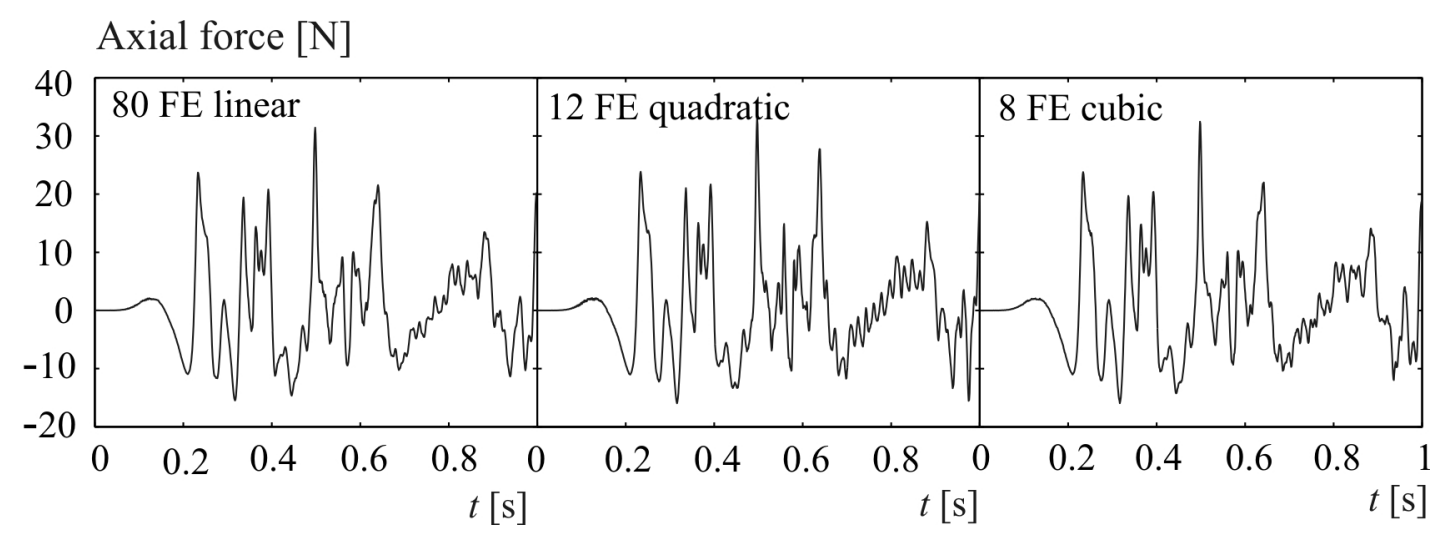

Fig. 9. Time variation of axial force at the first Gauss point to the left of the point of observation, $B$. Comparison of three different spatial interpolations.

demonstrate the advantage of the weak formulation in that it is capable of a much more stringent energy conservation. Without doubt this must help in 
long term calculations.

Table 2

Maximum difference in total energy in time interval $t \in[0.25,1]$. Different spatial interpolations, weak and strong variant.

\begin{tabular}{cccc}
\hline \multicolumn{4}{c}{$\max ($ Energy $)$-min (Energy) } \\
\hline & $80 \mathrm{FE} \mathrm{lin.}$ & $12 \mathrm{FE}$ quad. & $8 \mathrm{FE} \mathrm{cub}$. \\
\hline Weak formulation & $8.5 \cdot 10^{-8}$ & $7.7 \cdot 10^{-8}$ & $1.1 \cdot 10^{-7}$ \\
\hline Strong formulation & $9.5 \cdot 10^{-3}$ & $1.4 \cdot 10^{-4}$ & $1.4 \cdot 10^{-4}$ \\
\hline
\end{tabular}

The case is further analyzed, this time with an aim to compare the long term calculations. This time the problem is solved in $10 \mathrm{~s}$ interval. Vertical displacements at point $B$ as obtained by weak and strong formulations are shown in Fig. 10. The two solutions agree completely up to about $2.4 \mathrm{~s}$, and split into two separate curves afterwards.

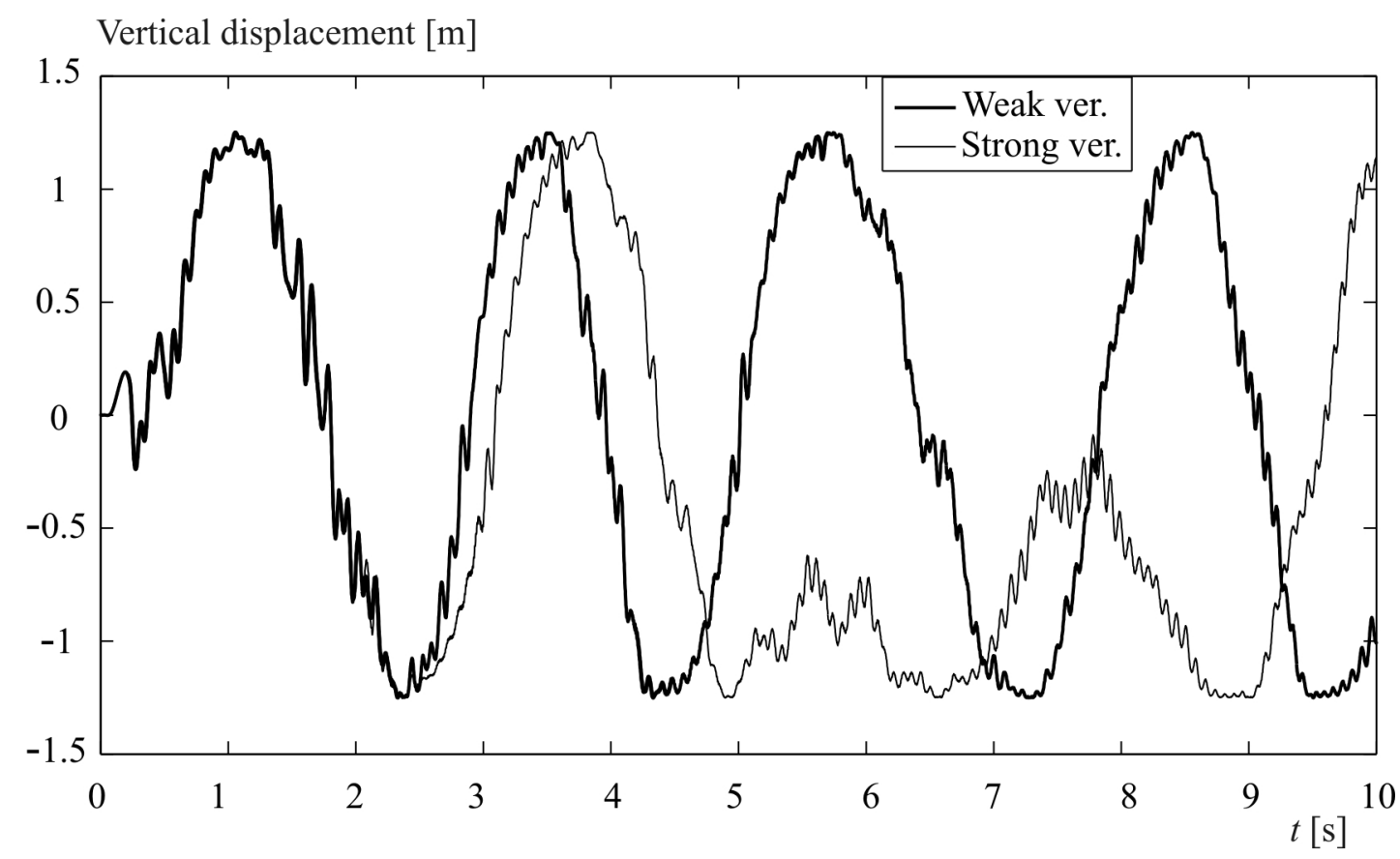

Fig. 10. Time variation of vertical displacement at point $B$. Comparison of results by weak and strong variants of the formulation. $12 \mathrm{FE}$ quadratic, $\Delta t=0.001 \mathrm{~s}$. 
A careful analysis reveals that the strong variant of the formulation experiences high frequency oscillations in vertical displacements at the instant of the split. These oscillations damp out in about $1 \mathrm{~s}$. No such phenomenon could be detected in the solution by the weak version. The solution by the strong version exhibits further unexpected behaviour after 5th second, which suggests that it has some sort of difficulties. The data in Table 3 show that the total energy is conserved with at least two orders of magnitude greater accuracy in the weak formulation, although the degree of conservation of the strong variant should also be considered sufficient to retain the stability of the time integration scheme.

Table 3

Maximum difference in total energy in time interval $t \in[0.25,10]$. Weak and strong variants of the formulation.

\begin{tabular}{cc}
\hline $\max ($ Energy $)-\min ($ Energy $)$ \\
\hline & $12 \mathrm{FE}$ quadratic \\
\hline Weak formulation & $1.7 \cdot 10^{-5}$ \\
\hline Strong formulation & $6.0 \cdot 10^{-3}$ \\
\hline
\end{tabular}

The graphs of total, kinetic and potential energies are shown in Fig. 11.

In order to asses the drift from the strain manifold due to the incrementaltype of the update, we computed the strains by Eqs. (2)-(4) once the updated displacements at $t_{n+1}$ have been evaluated, and made comparisons with the strains computed incrementally as given in Box 1. Small differences were found. This indicates that the drift is small and can be neglected.

The normalized errors of displacements at point $B$ and strains at the first Gauss point left to it, as a function of the number of time steps in the log$\log$ scale, are shown in Fig. 12. The error is calculated with respect to the 


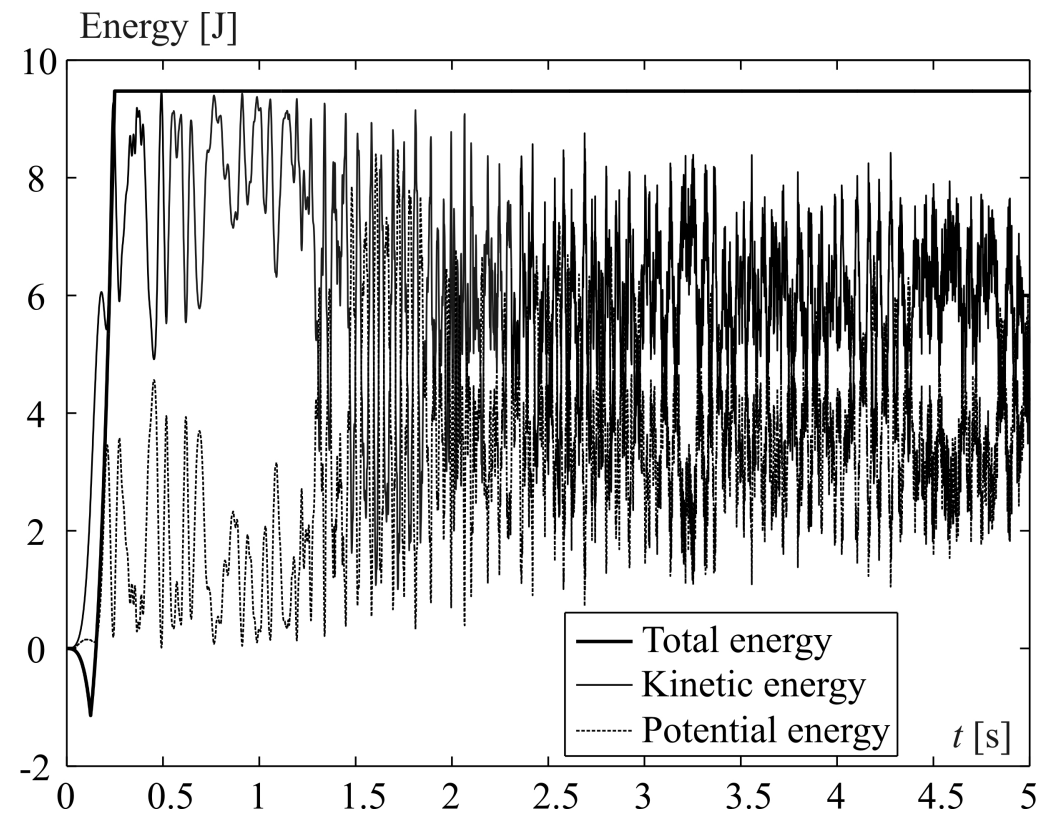

Fig. 11. Time variation of total, kinetic and potential energy of the system. Weak formulation. $16 \mathrm{FE}$ quadratic, $\Delta t=0.001 \mathrm{~s}$.

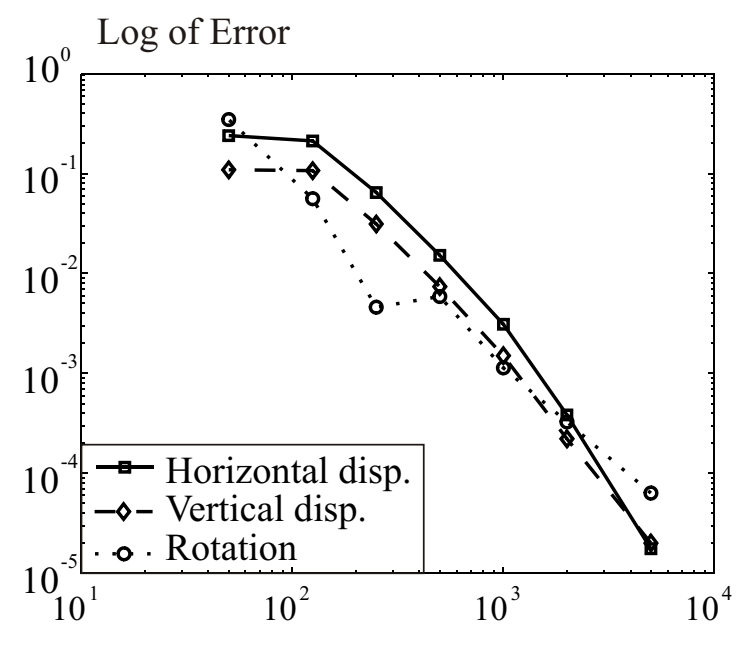

Log of Number of steps

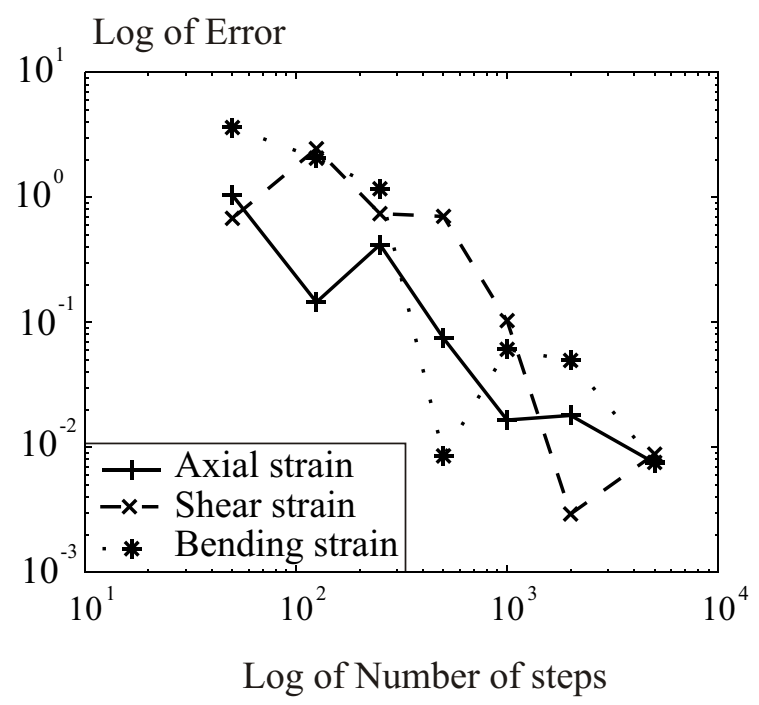

Log of Number of steps

Fig. 12. Convergence study.

reference solution at time $t=0.5 \mathrm{~s}$, and plotted as a function of the number of time steps. Analysis employs quadratic finite elements, and the reference solution uses $\Delta t=0.0000625 \mathrm{~s}$. The results show that the rate of convergence 
of the present time integration scheme combined with the quadratic elements is between two and three. Not much is changed if elements of various orders are employed, however.

\section{Conclusions}

A new time integration scheme has been presented for planar, elastic, geometrically exact beam-like structures, which perfectly conserves momenta and the total energy of the dynamic system with constant external loads. The scheme conserves the energy regardless of the particular spatial discretization chosen. It is distinct in that the unconventional, a finite-size incremental strain update is used, implied by a strict distinction between the infinitesimal and the finite change operator. The scheme is implemented in the displacement-based beam finite-element of geometrically exact theory of Reissner [30]. The automated code generation software package AceGen [25] was employed to generate the finite-element matrices needed for the computer code.

The energy conservation has been formally and numerically proved. Hence, the scheme is unconditionally stable [20]. The time discretization of the rotation matrix is assumed such that its orthogonality is retained. This is an advantage compared to the scheme proposed in [21-23] and many others.

The above scheme is marked the 'weak' scheme. In addition to the weak scheme, a 'strong' scheme has also been proposed which evaluates the current strains directly from the current generalized displacements. Such a 'strong' scheme does not conserve energy in an exact theoretical sense unless the updates for the velocity of rotation and for the torque are modified in a consistent manner [21]. 
Two well known numerical examples have been thoroughly analyzed. The results confirm a perfect conservation of energy for the weak formulation for any order of the spatial interpolation. A rather good, yet not perfect conservation of energy has been found when strong formulation is applied and the differences between the results were found to be very small. The investigation of the rate of convergence with regard to the time step size showed that the convergence order was between two and three.

Hence, the results of the numerical examples demonstrate an excellent performance in terms of the energy conservation, stability and accuracy of the scheme, and prove good convergence properties in terms of both the NewtonRaphson method and the time step size.

\section{Acknowledgment}

The work of M. Gams was supported by the Ministry of Higher Education, Science and Technology of the Republic of Slovenia under contract 8311-03831622. The support is gratefully acknowledged.

\section{References}

[1] F. Armero, I. Romero, "On the formulation of high-frequency dissipative timestepping algorithms for nonlinear dynamics. Part I: low-order methods for two model problems and nonlinear elastodynamics", Comput. Meth. Appl. Mech. Engrg. 190, 2603-2649, 2001.

[2] F. Armero, I. Romero, "On the formulation of high-frequency dissipative timestepping algorithms for nonlinear dynamics. Part II: second-order methods", Comput. Meth. Appl. Mech. Engrg. 190, 6783-6824, 2001. 
[3] O.A. Bauchau, G. Damilano, N.J. Theron, "Numerical integration of non-linear elastic multi-body systems", Int. J. Numer. Meth. Eng. 38, 2737-2751, 1995.

[4] O.A. Bauchau, N.J. Theron, "Energy decaying scheme for nonlinear elastic multi-body systems", Comput. Struct. 59, 317-331, 1996.

[5] O.A. Bauchau, N.J. Theron, "Energy decaying scheme for nonlinear beam model", Comput. Meth. Appl. Mech. Engrg. 134, 37-56, 1996.

[6] O.A. Bauchau, C.L. Bottasso, L. Trainelli, "Robust integration schemes for flexible multibody systems", Comput. Meth. Appl. Mech. Engrg. 192, 395-420, 2003.

[7] P. Betsch, P. Steinmann, "Inherently energy conserving time finite elements for classical mechanics", J. Comput. Phys. 160, 88-116, 2000.

[8] P. Betsch, P. Steinmann, "Constrained dynamics of geometrically exact beams", Comput. Mech. 31, 49-59, 2003.

[9] C.L. Bottasso, O.A. Bauchau, J.Y. Choi, "An energy decaying scheme for nonlinear dynamics of shells", Comput. Meth. Appl. Mech. Engrg. 191, 30993121, 2002.

[10] C.L. Bottasso, M. Borri, "Integrating finite rotations", Comput. Meth. Appl. Mech. Engrg. 164, 307-331, 1998.

[11] C.L. Bottasso, M. Borri, L. Trainelli, "Integration of elastic multibody systems by invariant conserving/dissipating algorithms. Part II: numerical schemes and applications", Comput. Meth. Appl. Mech. Engrg. 190, 3701-3733, 2001.

[12] Q.V. Bui, "Energy dissipative time finite elements for classical mechanics", Comput. Meth. Appl. Mech. Engrg. 192, 2925-2947, 2003.

[13] J. Chung, G. Hulbert, "A time integration algorithm for structural dynamics with improved numerical dissipation: the generalized $\alpha$ method", ASME J. Appl. Mech. 60:371-375, 1993.

[14] M.A. Crisfield, J. Shi, "A co-rotational element/time-integration strategy for non-linear dynamics”, Int. J. Numer. Meth. Eng. 37, 1897-1913, 1994.

[15] M.A. Crisfield, U. Galvanetto, G. Jelenić, "Dynamics of 3-D co-rotational beams", Comput. Mech. 20, 507-519, 1997.

[16] M. Gams, S. Srpčič, M. Saje, I. Planinc, "Finite element dynamic analysis of geometrically exact beams", accepted Comput. Struct.

[17] M. Géradin, A. Cardona, "Flexible Multibody Dynamics. A Finite Element Approach", John Wiley and Sons Ltd, 2001.

[18] J.M. Goicolea, J.C.G. Orden, "Quadratic and higher-order constraints in energy-conserving formulations of flexible multibody systems", Multibody Sys. Dyn. 7, 3-29, 2002. 
[19] H.M. Hilber, T.J.R. Hughes, R.L. Taylor, "Improved numerical dissipation for time integration algorithms in structural dynamics", Earthquake Eng. Struct. Dyn. 5, 282-292, 1977.

[20] T.J.R. Hughes, "The Finite Element Method", Prentice Hall, Englewood Cliffs, N.J., 1992.

[21] A. Ibrahimbegović, S. Mamouri, "Nonlinear dynamics of flexible beams in planar motion: formulation and time-stepping scheme for stiff problems", Comput. Struct. 70, 1-22, 1999.

[22] A. Ibrahimbegović, S. Mamouri, "Energy conserving/decaying implicit timestepping scheme for three-dimensional beams undergoing finite rotations", Comput. Meth. Appl. Mech. Engrg. 191, 4241-4258, 2002.

[23] A. Ibrahimbegović, S. Mamouri, R.L. Taylor, A.J. Chen, "Finite element method in dynamics of flexible multibody systems: Modeling of holonomic constraints and energy conserving integration schemes", Multibody Sys. Dyn. 4, 195-223, 2000 .

[24] G. Jelenić, M.A. Crisfield, "Dynamic analysis of 3D beams with joints in presence of large rotations", Comput. Meth. Appl. Mech. Engrg. 190, 41954230, 2001.

[25] J. Korelc, "Automatic generation of finite-element code by simultaneous optimization of expressions", Theor. Comput. Sci. 187, 231-248, 1997.

[26] D. Kuhl, M.A. Crisfield, "Energy conserving and decaying algorithms in nonlinear structural dynamics", Int. J. Num. Methods in Eng. 45, 569-599, 1999.

[27] D. Kuhl, E. Ramm, "Generalized energy-momentum method for non-linear adaptive shell dynamics", Int. J. Num. Methods in Eng. 178, 343-366, 1999.

[28] MATLAB, "Using MATLAB, the language of technical computing", The MathWorks, Inc., Natick, MA, 1999.

[29] N.M. Newmark, "A method of computation for structural dynamics", J. Eng. Mech. Div. ASCE 85, 67-94, 1959.

[30] E. Reissner, "On one-dimensional finite-strain beam theory: the plane problem", J. Appl. Math. Physics (ZAMP) 23, 795-804, 1972.

[31] I. Romero, F. Armero, "Numerical integration of the stiff dynamics of geometrically exact shells: an energy-dissipative momentum-conserving scheme", Int. J. Num. Methods in Eng. 54, 1043-1086, 2002.

[32] M. Saje, I. Planinc, G. Turk, B. Vratanar, "A kinematically exact finite element formulation of planar elastic-plastic frames", Comput. Meth. Appl. Mech. Engrg. 144, 125-151, 1997.

[33] C. Sansour, P. Wriggers, J. Sansour, "Nonlinear dynamics of shells: Theory, finite element formulation, and integration schemes", Nonlinear Dyn. 13, 279305, 1997. 
[34] C. Sansour, W. Wagner, P. Wriggers, J. Sansour, "An energy-momentum integration scheme and enhanced strain finite elements for the non-linear dynamics of shells", Int. J. Non-lin. Mech. 37, 951-966, 2002.

[35] J.C. Simo, L. Vu-Quoc, "On the dynamics of flexible beams under large overall motions - The plane case: Part I and Part II", ASME J. Appl. Mech 53, 849$854,1986$.

[36] J.C. Simo, N. Tarnow, M. Doblare, "Non-linear dynamics of three-dimensional rods: exact energy and momentum conserving algorithms", Z. angew. Math. Phys. (ZAMP) 43, 757-793, 1995.

[37] J.C. Simo, N. Tarnow, "The discrete energy-momentum method. Conserving algorithms for nonlinear elastodynamics", Int. J. Numer. Meth. Eng. 38, 14311473, 1995.

[38] N. Stander, E. Stein, "An energy conserving planar finite beam element for dynamics of flexible mechanisms", Eng. Comput. 13(6), 60-85, 1996.

[39] S. Wolfram, "Mathematica", Addison-Wesley Publishing Company, Inc., 1991. 